\title{
Commentaries
}

\section{Mind over matter in the postinfective irritable bowel}

In recent years, our understanding of the irritable bowel syndrome (IBS) has evolved from being considered solely a disorder of motility, to one that is characterised by dysregulation of brain-gut function, manifest by gut motor hyper-reactivity and enhanced visceral sensitivity to various stresses. ${ }^{1}$ In this regard, several psychosocial factors, namely life stress, abuse, psychological co-morbidity, and illness behaviour, have been implicated in the clinical expression of IBS. ${ }^{2}$ However, although most published studies have shown an association of psychosocial factors with IBS, only one study until now, has reported that psychosocial distress in patients with acute infectious diarrhoea predicted the later development of IBS symptoms. ${ }^{3}$ In a follow up study in this issue, Gwee et al (see page 400) elaborate upon this observation by describing the psychosocial, physiological and histopathological features of individuals who develop postinfectious IBS symptoms.

In a sample of 94 patients with acute gastroenteritis studied in an infectious disease unit, 22 (23\%) were found three months later to fulfil Rome criteria for IBS. ${ }^{4}$ When comparing this group of symptomatic patients (IBS+) to the remaining sample $(77 \%)$ who resolved their gut symptoms (IBS-), three major findings were noted. (1) Psychosocial difficulties were again shown to influence who with acute gastroenteritis would remain symptomatic. Specifically, in a regression analysis, pre-existent life stress and hypochondriasis were the strongest predictors of the IBS+ group, and the development of IBS-like symptoms was not explained by illness behaviours. (2) Acute gastroenteritis was associated with increased numbers (relative to biopsy samples taken from a healthy control group) of rectal inflammatory cells. However, three months later, only the IBS+ group continued to have increased mucosal inflammation. (3) When compared with healthy controls, those with the acute gastroenteritis went on at three months to develop physiological evidence of gut dysfunctionreduced whole gut transit time, decreased sensation threshold to rectal distension, decreased rectal compliance and reduced number of rectal contractions. However, there were no significant differences physiologically between the IBS+ and IBS - groups.

One conclusion noted by the authors harmonises with a biopsychosocial understanding of IBS $^{5}$ : “. . . physiological changes predispose to IBS, but psychological factors are required to bring out the perception of these physiological changes as symptoms." So, the presence of dysmotility or visceral hypersensitivity is not sufficient to explain fully the symptoms of IBS; the individual's psychosocial state must also be considered. Another conclusion, that psychosocial distress may perpetuate gut inflammation, offers relatively new information that needs elaboration with regard to IBS. Presumably this may occur via psychoimmunological mechanisms that converge on neuropeptide function in the gut lamina propria. ${ }^{6}$ In a study on rats, previous inflammation upregulated or rekindled the host's inflammatory response to later stress. ${ }^{6}$ Wistar-Kyoto and Sprague-
Dawley rats that recovered from experimental colitis induced by trinitrobenzene sulphonic acid when subsequently stressed by physical restraint, showed a fourfold increase in tissue myeloperoxidase activity, a measure of tissue inflammatory response. Once inflammation exists in the gut, the activation of local cytokines and possibly other mechanisms may induce hypermotility and enhanced visceral sensitivity even after the inflammation subsides.

Figure 1 conceptualises possible associations relating inflammation, psychosocial distress, physiological dysfunction, and symptoms in individuals with postinfectious gastrointestinal symptoms. Firstly, an acute enteric infection will lead to mucosal inflammation and dysmotility (either directly or via inflammatory effects). Secondly, mucosal inflammation or dysmotility, or both, may eventually lead to visceral hypersensitivity - that is, low thresholds to rectal distension. ${ }^{8}$ Thirdly, IBS-like symptoms may result from visceral hypersensitivity in the gut and dysmotility. However, in Gwee et al's study, these gut related physiological factors were not sufficient to explain the IBS symptoms as they were also present in the postinfectious group without IBS symptoms. Thus, the study suggests that psychosocial factors may have an overarching effect on symptom development in postinfectious IBS. This may occur through putative effects on mucosal inflammation (and inflammation may have reciprocal effects on psychological state via activation of peripheral or central, or both, cytokines ${ }^{9}$ ), through central influences on peripheral visceral sensitisation or dysmotility (via descending corticofugal systems), or by direct effects on IBS symptoms via central nervous system modulation of incoming afferent input.

There are several limitations in this study which may affect the interpretation of the results and the conclusions drawn from the data. Firstly, postinfectious gut dysfunction may not actually be IBS, or it may represent only a small subset of patients with IBS. Three months is at the lower time limit for diagnosing IBS based on Rome criteria, ${ }^{4}$ and it is uncertain what proportion of patients with symptoms three months after enteric infection would still be symptomatic six or 12 months later. Furthermore, the proportion of patients with constipation-like IBS is not reported; it is likely that patients with postinfectious bowel dysfunction would primarily have symptoms of diarrhoea. A second limitation relates to a possible acquisition bias. In addition to the 94 patients studied at intake and three months, 15 other patients initially evaluated did not return for the three month follow up tests. Furthermore, although

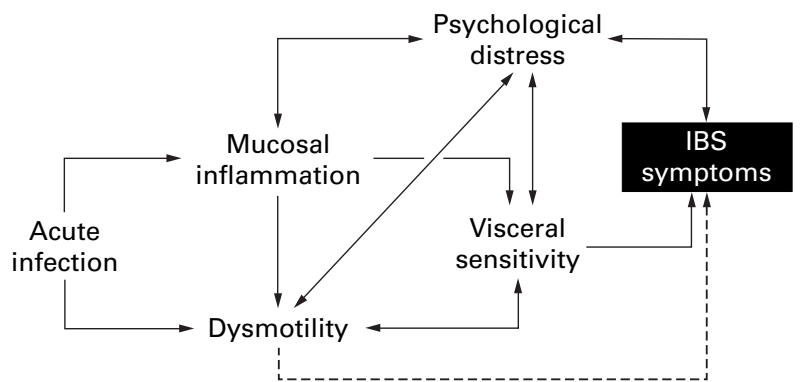

Figure 1 Possible associations relating inflammation, psychosocial distress, physiological dysfunction, and symptoms in individuals with postinfectious gastrointestinal symptoms. 
all patients were invited for histopathological and physiological investigation, only $10(45 \%)$ of 22 IBS + and 19 $(26 \%)$ of 72 IBS - patients had rectal biopsy, and $16(73 \%)$ of 22 IBS + and $16(22 \%) 72$ IBS- patients underwent physiological testing. So it is possible that the relatively small proportion of patients tested are not truly representative of the total population under question. Thirdly, almost half of the patients did not have a positive stool culture for enteric pathogens. Although many enteric infections are culture negative, for the purpose of a clinical research study, the possibility exists that a substantial proportion of the patients may in fact have had IBS in an early clinical presentation. A final consideration relates to whether increased rectal inflammation is typical for IBS. In one study, comparing 61 patients fulfilling Rome criteria for IBS with 59 controls, ${ }^{10}$ there was no difference between groups with regard to histopathology on rectal biopsy, and none of the patients had evidence of microscopic colitis. Although quantitative cell counts were not done, there is little qualitative evidence for increased levels of mucosal inflammation in IBS. We also need to question whether the inflammation resulted from patient related behaviours. For example, would a psychologically distressed individual having symptoms be more likely to take aspirin or non-steroidal medication, thereby increasing the likelihood of having mucosal inflammation?

These limitations are likely to be tackled in future studies by the authors and other investigators. What is important is that the findings are new and provocative. They lead us to consider additional questions for future study. (1) Are there pre-morbid psychosocial difficulties that predict who will develop an enteric infection, or does an infection unmask patients having high susceptibility to respond with psychological distress? This question could only be answered by studying patients psychosocially before the inoculation of the enteric infection. (2) What is the role for gut motor dysfunction in the symptoms of IBS? The evidence in this study suggests that dysmotility does not relate to symptoms directly, but rather develops in response to the infection. Future studies may determine whether gut motor dysfunction has indirect effects on symptoms, possibly by influencing the development of visceral hypersensitivity. Finally, (3) in the psychologically susceptible individual, would early psychopharmacological (or psychological) treatment prevent the full blown development of IBS (e.g. by reducing mucosal inflammation or visceral sensitisation)?

UNC Functional GI Disorders Centre

D A DROSSMAN

Division of Digestive Diseases,

726 Burnett-Womack Bldg. CB 7080,

University of North Carolina,

Chapel Hill, N.C. 27599-7080, USA

1 Drossman DA. The functional gastrointestinal disorders and their diagnosis: a coming of age. In: Drossman DA, Richter JE, Talley NJ, et al, eds. The functional gastrointestinal disorders: diagnosis, pathophysiology and treatment. McLean, VA: Degnon Associates, 1994:1-23.

2 Drossman DA, Creed FH, Fava GA, et al. Psychosocial aspects of the functional gastrointestinal disorders. Gastroenterol Int 1995;8:47-90.

3 Gwee KA, Graham JC, McKendrick MW, et al. Psychometric scores and persistence of irritable bowel after infectious diarrhoea. Lancet 1996;347: 150-3.

4 Thompson WG. C. Functional bowel disorders and D. Functional abdominal pain. In: Drossman DA, Richter JE, Talley NJ, et al, eds. The functional gastrointestinal disorders: diagnosis, pathophysiology and treatment. McLean, VA: Degnon Associates, 1994:115-73.

5 Drossman DA. Presidential address: Gastrointestinal illness and biopsychosocial model. Psychosom Med 1998;60:258-67.

6 Collins SM, McHugh K, Jacobson K, et al Previous inflammation alters the response of the rat colon to stress. Gastroenterology 1996;111:1509-15.

7 Collins SM. The immunomodulation of enteric neuromuscular function: Implications for motility and inflammatory disorders. Gastroenterology

8 Mayer EA, Gebhart GF. Basic and clinical aspects of visceral hyperalgesia. Gastroenterology 1994;107:271-93.

9 Sternberg EM. Perspectives series: Cytokines and the brain. $\mathcal{F}$ Clin Invest 1997;100:2641-7.

10 MacIntosh DG, Thompson WG, Patel DG, et al. Is rectal biopsy necessary in irritable bowel syndrome? Am f Gastroenterol 1992;87:1407-9.

\section{CagA, the cag pathogenicity island and Helicobacter pylori virulence}

Most people with stomachs colonised by Helicobacter pylori do not develop peptic ulceration, gastric adenocarcinoma or gastric lymphoma in their lifetime. Development of disease is likely to be determined by a combination of factors: the virulence of the infecting $H$ pylori strain, the susceptibility of the host and environmental co-factors. The best studied of these disease determinants is strain virulence, and the two most studied virulence markers are expression of vacuolating cytotoxin activity and production of a non-toxigenic protein of unknown function called CagA. ${ }^{1}$ Colonisation with CagA+ strains of $H$ pylori is easy to detect as CagA provokes a strong systemic antibody response in the human host. In Europe and the USA, more than $60 \%$ of those colonised by $H$ pylor $i$ have antibodies to CagA: these people have more intense gastric inflammation than those colonised by CagA- $H$ pylori alone and are more likely to develop peptic ulcers and distal gastric adenocarcinoma. ${ }^{1}$ However, most people colonised by CagA + strains do not develop these conditions and it may be more useful to regard CagA- strains as having reduced pathogenicity than to regard CagA+ strains as pathogenic. In Japan, where Maeda et al's study (see page 336) was conducted, not only is $H$ pylori colonisation more prevalent than in the West, but also colonisation by CagA- strains is rare. ${ }^{2} 3$ Both of these factors may contribute to the high prevalence of $H$ pylori associated disease in Japan.

Advances in $H$ pylori genetics have led to a better understanding of why CagA+ strains cause more inflammation and disease than CagA- strains. The CagA protein is named after the gene encoding it, cytotoxin-associated gene $A(\operatorname{cag} A) \operatorname{cag} A$ is misleadingly named, in that it does not encode the cytotoxin (the cytotoxin gene is vacA) and its artificial disruption does not affect cytotoxin production or activity. ${ }^{45}$ However, mapping of the $H$ pylori chromosome around $\operatorname{cag} A$ has shown that it is at one end of a group of about 30 genes which have a different nucleotide content to the rest of the chromosome. This has features of a pathogenicity island (PAI), a group of genes acquired by a bacterium relatively recently in its evolution which render it more pathogenic, ${ }^{6}$ and has been named the cag PAI. ${ }^{7}$ The cag PAI, but not cag $A$ itself, is thought to be important for the enhanced inflammation associated with CagA+ strains. The evidence for this is that individual artificial disruption of many cag PAI genes (although not $\operatorname{cag} A$ ) reduces the ability of $H$ pylor $i$ to induce cultured epithelial cells to release the pro-inflammatory cytokine interleukin $8 .^{7-9}$ Exactly what the cag PAI encodes is still unclear, but comparisons with similar genes in other bacteria suggest that it may be a secretion system for other proteins or a mechanism for interacting closely with epithelial cells. ${ }^{7-9}$ The cytotoxin gene, vacA, is not on the cag PAI and the reason for the close genetic association between certain 
types of $v a c A$ and the presence of the cag PAI is still unknown. ${ }^{10}$

The paper by Maeda et al assesses the extent to which production of the CagA protein or possession of the $\operatorname{cag} A$ gene is a marker for the presence of an intact cag PAI in Japanese strains. It confirms the high prevalence of cag $A+$ strains in Japan (100\% in this study) and thus the low prevalence of $c a g A-$ strains compared with Europe and the USA. Among the $\operatorname{cag} A+$ strains, six of 63 did not produce the CagA protein, a similar proportion to that found in a European study. ${ }^{5}$ Two of these strains had an intact cag PAI, induced enhanced interleukin 8 release from epithelial cells and were isolated from patients with peptic ulcers, suggesting that they were fully pathogenic strains. The other four had sections of the cag PAI missing, induced low levels of interleukin 8 production and were isolated from patients without ulcers, suggesting that they had reduced pathogenicity. For one of these four strains with partial cag PAI deletions, the reason for lack of $\operatorname{cag} A$ expression was sought: it was found that the missing section of the cag PAI included the promoter and 5 ' end of $\operatorname{cag} A$, rendering the gene non-functional. Although numbers are small, data on these strains are consistent with the hypothesis that enhanced pathogenicity is dependent on the presence of an intact cag PAI.

The authors further explored the mechanism underlying cag PAI partial deletions by testing for the presence of insertion sequence (IS) 605, a stretch of DNA which may be present anywhere in the $H$ pylori chromosome and is thought to be involved in rearranging the order of $H$ pylori genes. It has been hypothesised that IS 605 was acquired by $H$ pylori later than the cag PAI in evolutionary time and is a prerequisite for cag deletions. ${ }^{7}$ Maeda et al found that two of the four strains with partial cag PAI deletions lacked IS605 and repeated the observation that some Western strains lacking the cag PAI possessed IS605. Although not disproving the original explanation of cag deletion, these findings suggest that deletion without IS605 may be possible and further research is needed to clarify this. Particular interest surrounds whether cag deletion is an ongoing phenomenon, perhaps allowing better co-adaption between $H$ pylori and its host, or whether the deletion events occurred distantly in evolutionary time. Further characterisation of the strains in this study may help address this point, especially if serum samples from the hosts are also available.
The authors' main conclusion is that neither the $\operatorname{cag} A$ gene nor the CagA protein are invariable markers of the intact cag PAI. From their data on Japanese strains, other cag genes may be better markers, although whether this will be the case in different populations remains to be determined. In practical terms, serological testing is needed for large studies and whether these other cag genes encode sufficiently immunogenic proteins for this to be possible is unknown. The current study does not assess the accuracy of serological testing for CagA, but it is reassuring that 57/59 cag PAI+ strains were CagA+ (and so would be expected to provoke an antibody response) and that all four strains with cag PAI partial deletions were CagA- (and so would be expected not to provoke such a response). In the West, colonisation by CagA- strains, most of which lack cag $A$ and the whole cag PAI, also produces CagA negative serological tests. ${ }^{57}$ Thus, although the case is not completely proved, a positive serological test for CagA is likely to be a reasonable marker for colonisation with an $H$ pylori strain possessing an intact cag PAI.

Division of Gastroenterology and

J C ATHERTON

Institute of Infections and Immunity,

University Hospital,

Nottingham NG7 2 UH, UK

1 Atherton JC. H. pylori virulence factors. Br Med Bull 1998;54(No.1):105-20. 2 Shimoyama T, Fukuda S, Tanaka M, et al. High prevalence of the CagA-positive Helicobacter pylori strains in Japanese asymptomatic patients and gastric cancer patients. Scand f Gastroenterol 1997;32:465-8.

3 Maeda S, Ogura K, Yoshida $\mathrm{H}$, et al. Major virulence factors, VacA and CagA, are commonly positive in Helicobacter pylori isolates in Japan. Gut 1998;42:338-43.

4 Tummuru MKR, Cover TL, Blaser MJ. Mutation of the cytotoxinassociated $\operatorname{cag} A$ gene does not affect the vacuolating cytotoxin activity of Helicobacter pylori. Infect Immun 1994;62:2609-13.

5 Xiang, Z, Censini S, Bayeli PF, et al. Analysis of expression of CagA and VacA virulence factors in 43 strains of Helicobacter pylori reveals that clinical isolates can be divided into two major types and that CagA is not clinical isolates can be divided into two major types and that CagA is not 1995;63:94-8.

6 Hacker J, Blum-Oehler G, Muhldorfer I, et al. Pathogenicity islands of virulent bacteria: structure, function and impact on microbial evolution. $\mathrm{Mol}$ Microbiol 1997;23:1089-97.

7 Censini S, Lange C, Xiang Z, et al. cag, a pathogenicity island of Helicobacter pylori, encodes type I-specific and disease-associated virulence factors. Proc Natl Acad Sci USA 1996;93:14648-53.

8 Akopyantz NS, Clifton SW, Kersulyte D, et al. Analysis of the cag pathogenicity island of Helicobacter pylori. Mol Microbiol 1998;28:37-53.

9 Tummuru MKR, Sharma SA, Blaser MJ. Helicobacter pylori picB, a homologue of the Bordetella pertussis toxin secretion protein, is required for induction of IL-8 in gastric epithelial cells. Mol Microbiol 1995;18:867-76.

10 Atherton JC, Cao P, Peek RM, et al. Mosaicism in vacuolating cytotoxin alleles of Helicobacter pylori: association of specific vacA types with cytotoxin production and peptic ulceration. F Biol Chem 1995;270:17771-7. 